LA W RENCE LIVERMORE N A T IO N A L LABORATORY

\section{Babel 1.0 Release Criteria: A Working Document}

Gary Kumfert, Tamara Dahlgren, Thomas Epperly, James Leek

October 21, 2004 
This document was prepared as an account of work sponsored by an agency of the United States Government. Neither the United States Government nor the University of California nor any of their employees, makes any warranty, express or implied, or assumes any legal liability or responsibility for the accuracy, completeness, or usefulness of any information, apparatus, product, or process disclosed, or represents that its use would not infringe privately owned rights. Reference herein to any specific commercial product, process, or service by trade name, trademark, manufacturer, or otherwise, does not necessarily constitute or imply its endorsement, recommendation, or favoring by the United States Government or the University of California. The views and opinions of authors expressed herein do not necessarily state or reflect those of the United States Government or the University of California, and shall not be used for advertising or product endorsement purposes.

This work was performed under the auspices of the U.S. Department of Energy by University of California, Lawrence Livermore National Laboratory under Contract W-7405-Eng-48. 


\title{
Babel 1.0 Release Criteria: A Working Document
}

\author{
Gary Kumfert, Tamara Dahlgren, Thomas Epperly, James Leek
}

December 2003

\author{
(updated October 2004 after version 0.9.6)
}

\section{Overview}

In keeping with the Open Source tradition, we want our Babel 1.0 release to indicate a certain level of capability, maturity, and stability. From our first release (version 0.5 .0 ) in July of 2001 to our current $\left(18^{\text {th }}\right)$ release (version 0.9 .6 ) we have continued to add capabilities in response to customer feedback, our observations in the field, and a consistent vision for interoperability. The key to our maturity is without a doubt the ever-increasing demands of our growing user base... both in terms of sheer size and sophistication with the underlying technology.

Stability is a special challenge for any research project. With our 1.0 release, we will branch and maintain a stable Babel 1.0 code line for at least a full year. This means no new features and no backward incompatible changes, only bug fixes. All continuing R\&D will be performed on a separate development tree. Currently, Babel has a quarterly release cycle with no guarantee for backward compatibility from one release to the next (though we certainly try to make migration as painless as possible).

Now is the time where we can see a good point for a Babel 1.0 release. But, seeing that point is different from being there. This list enumerates and explains the outstanding technical issues to be resolved to minimize volatility and help ensure stability for the 1.0 line.

The first draft of this document was circulated internally in June 2003. A revised draft was then presented at the July 2003 CCA meeting. A third revision was made into the current working document form \& circulated for general comment on the babel-users mailing list and Babel's homepage. The working document was intended to be an open record tracking progress in subsequent Babel releases. A major revision of the document (including adding new items and promoting/demoting itmes) was done in October 2004, well after the 0.9.6 release. 


\section{Table of Contents}

To-Do Items for $1.0 \ldots \ldots \ldots \ldots \ldots \ldots \ldots \ldots \ldots \ldots$

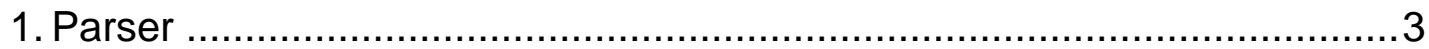

a. Regularize Type Resolution Behavior ..............................................

b. Allow XML overwrites (added Oct 2004)......................................... 3

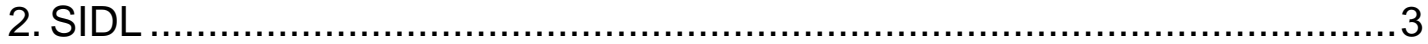

a. Add global scope indicator (.) (like leading :: in C++) ..........................3

b. Resolve issues with multiple inheritance induced overloading ...............4 4

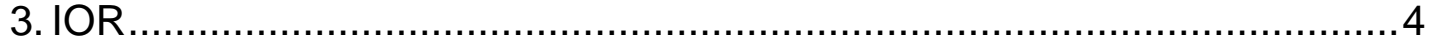

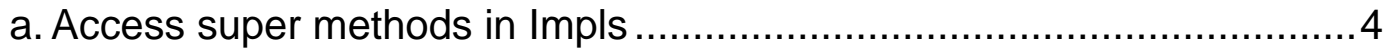

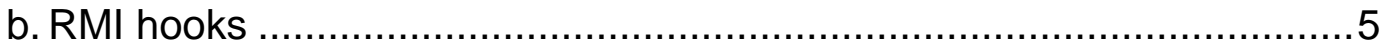

c. Pre/post method hooks............................................................. 5

d. Static Class Initializer a.k.a. "_load()" (added Oct 2004)......................5

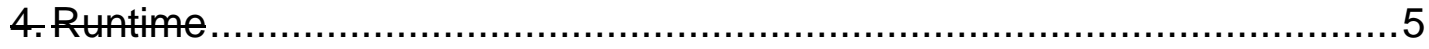

a. Better shared library lookup in SIDL.Loader ..................................... 5

b. Move Base Classes from "SIDL" package to "sidl" package .................... 6

c. Change SIDL.BaseException from a class to an Interface ....................6

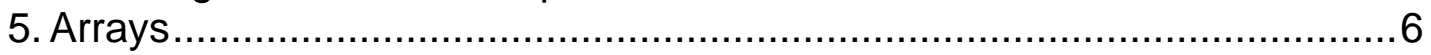

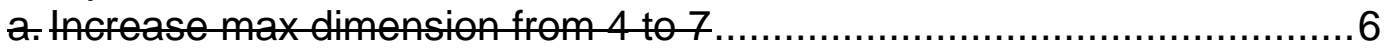

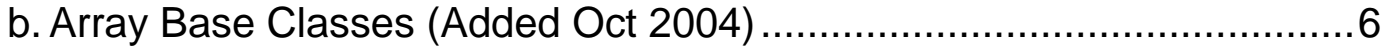

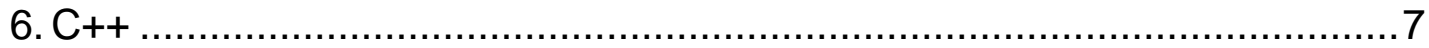

a. Resolve issues with overloading based on object type ......................... 7

b. Resolve issues with exception handling of Babel smart-pointers ........... 7

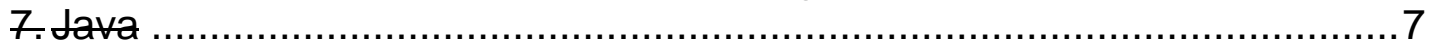

a. Implement Array support (client and server) .................................... 7

b. Implement support for Objects as arguments (client and server) .......... 7

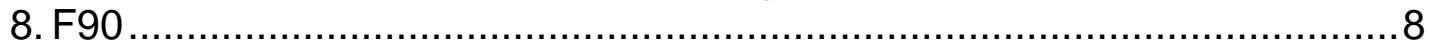

a. Provide native access to Babel arrays (a.k.a. Phase III).......................8

b. Find solution for compilers that treat intrinsic functions as reserved

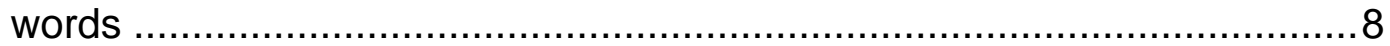

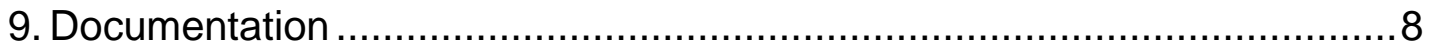

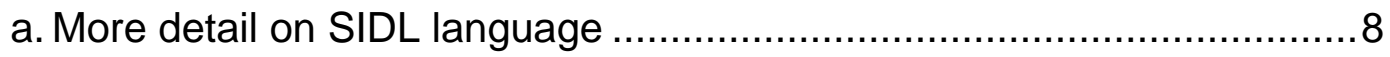

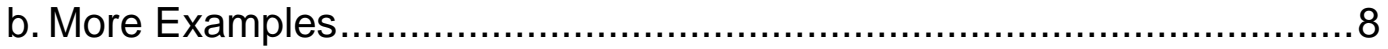

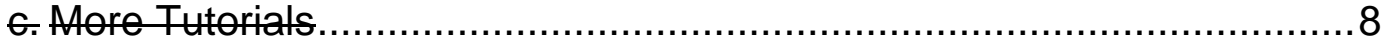

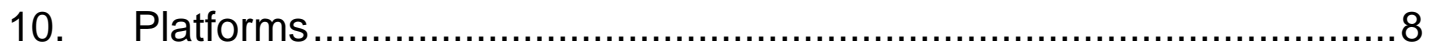

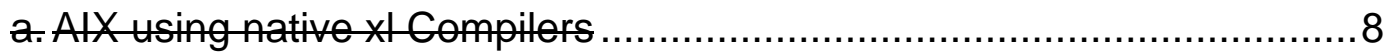

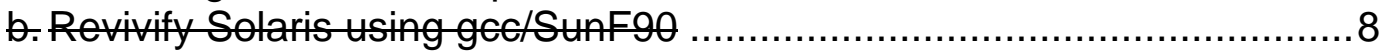

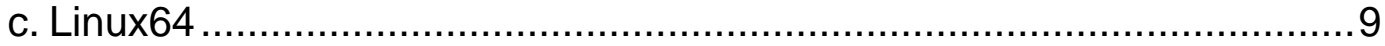

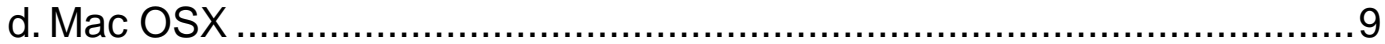

Deferred Items ........................ 10 


\section{To-Do Items for 1.0}

\section{Parser}

\section{a. Regularize Type Resolution Behavior}

The Babel parser currently is too aggressive in resolving user-defined types.
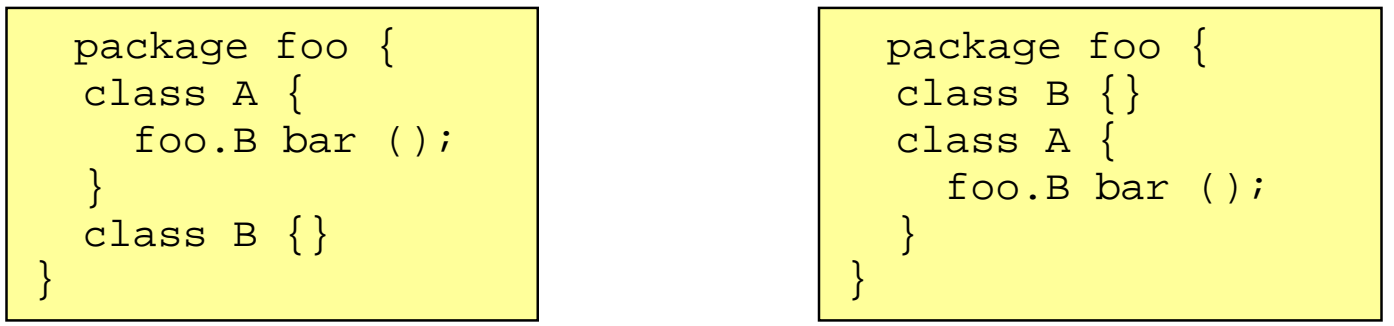

This currently results in problems of SIDL files needing to be specified in particular orders and the two samples above resolving the return type of the function foo.A.bar() differently. Clearly a lazy resolution technique is needed where resolution phase is distinct from initial parsing.

\section{b. Allow XML overwrites (added Oct 2004)}

Sometimes users wish to refresh XML files based on modifications to their SIDL sources. If the target XML files are co-located with other XML files that are required for type resolution, then it is logical to use $-\mathrm{O}$ and $-\mathrm{R}$ flags pointing to same repository. In this case, Babel will generate symbol redefinition errors and does not have a flag to force overwrites.

\section{2. $\quad S I D L$}

Every SIDL grammar change listed is an addition to the current SIDL spec. Therefore, all existing SIDL files will be valid even after the changes are released.

\section{a. Add global scope indicator (.) (like leading :: in C++)}

There are limited cases where presented with multiple options, SIDL gives no

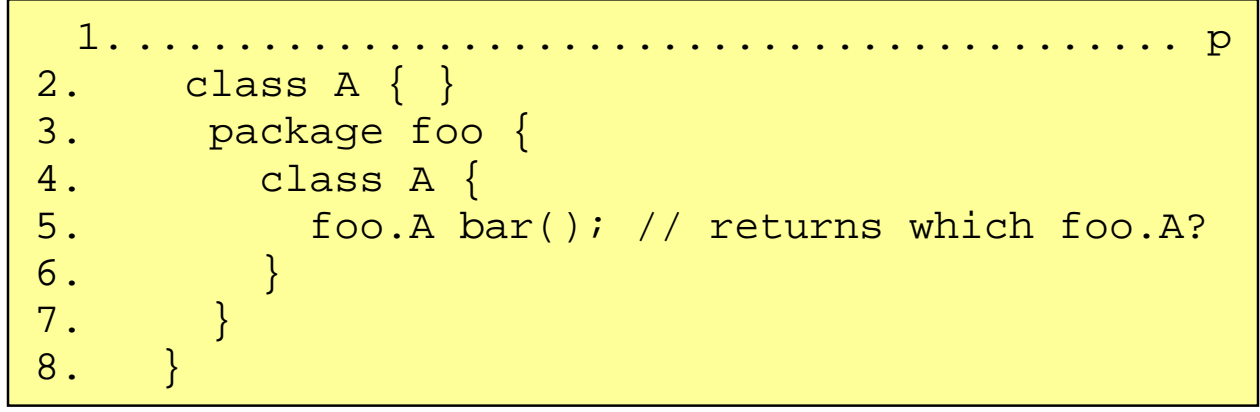

easy way to specify exactly which choice the user wants. In the SIDL example above, line 5 shows a method bar() that returns a foo.A. Currently, 
this resolves to the class in the same package, and its not possible to refer to the class $\mathrm{A}$ in the outermost package. Adding a leading dot (.) to the syntax resolves this issue. The user could specify ".foo.A" to resolve the ambiguity.

\section{b. Resolve issues with multiple inheritance induced overloading}

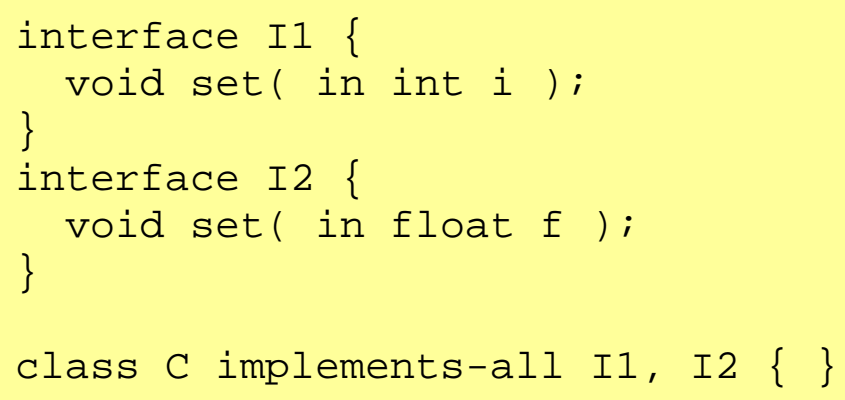

The SIDL fragment above is currently invalid. The multiple inheritance of interfaces would be fine if both set() methods had the same signature. However, since they are different and since they are inherited from different interfaces they are not overloaded. SIDL's grammar and Babel's parser need to be augmented to cover this case.

Tentatively the new syntax would look like the following SIDL fragment.

class C implements I1, I2 \{ void set[Int](in int i) = I1. set;

\} void set[Flt](in float $f$ ) = I2. set;

Here, the equals operator allows us to redefine the name of a method that is inherited, but does not allow the signature to be changed.

\section{IOR}

Because the IOR is the key to Babel's interoperability, we expect each change listed here to be non-backward compatible with older IORs. Customers will have to run the newer Babel code generator over their older SIDL files and Impls to generate new IORs.

\section{a. Access super methods in Impls}

Often times in the implementation of a method, it is common to want to call the parent class's implementation. Babel currently stores the Entry Point Vector (EPV) for the parent class as part of proper construction/destruction, but the EPV is hidden in a static variable in the IOR.c file, and thereby not exposed for the implementation to use. 
We need to provide some equivalent to "super" methods in Java. Super indicates methods in the super class (parent class) and is only available within the implementation of the derived class.

As of December 2003, this activity is in the initial design stage. As of August 2004, work on the IOR is complete, and changes to all language bindings are underway.

\section{b. RMI hooks}

Babel will define a SIDL interface standard for Remote Method Invocation (RMI) and will generate hooks in the IOR to call those interfaces. This will allow interested researchers with communication libraries to implement these interfaces and plug their code into Babel.

As of December 2003, there are some hand-generated prototypes using Ken Chiu's Proteus multi-protocol library from Indiana University.

\section{c. Pre/post method hooks}

There are many cases where one may want to hook arbitrary code as a precondition or postcondition of a Babel method invocation. Examples include logic checking, timer insertion, flow traces, and QoS. Babel will define and implement a general standard to satisfy the community's interest in this feature.

As of December 2003, we have received requirements from the TAU team for instrumenting Babel code with timers. A draft proposal is in the design stages and will be circulated to the babel-users list for comment.

\section{d. Static Class Initializer a.k.a. “_load()” (added Oct 2004)}

Create a user-defined method in all Impls that is guaranteed to be (1) envoked at most once, and (2) envoked before any other user-defined code (including static methods or constructors). This is useful (among other things) for initializing singleton classes that have all static method accessors.

\section{Runtime}

\section{$a_{\text {. }}$ Better shared library lookup in SIDL.Loader}

When asked to load a symbol at runtime, the original SIDL.Loader would go through its path, recursively opening every so file looking for the symbol in question. If the user set their SIDL_DLL_PATH to “”, the SIDL.Loader would find it... eventually. The problems with this all too permissive approach were (1) it was hard to debug, (2) it would try to open things that had a so extension, but were not made to be dynamically loaded. (3) it was too hard to control. 
The SIDL.Loader implementation will be rewitten to be must less pemissive and easier to control and debug. The new implementation will rely on auxiliary XML files to specify exactly what symbols are to be found in what libraries.

As of December 2003, the SIDL.Loader is currently implemented and introduces non-backward compatible changes. It will be released in the upcoming Babel 0.9.0 release.

\section{b. Move Base Classes from "SIDL" package to "sid"" package}

Unfortmnately some $\mathrm{C} / \mathrm{C}++$ header files on some architectures \#define SIDL to be 4 . This causes problems in $\mathrm{C}++$ header files that put everything in the namespace SIDL. We have always recommended that SIDL package names be all in lowerease, but for historical reasons haven't followedour own advice. Since the convention is that preprocessor macros are always all uppercase, we are resolved to rename the SIDL package to lowercase.

This change should avoid preprocessor problems, but will introduce a nonbackward compatible change when released. As of December 2003, there has been no work on this item.

\section{Change SIDL.BaseException from a class to an Interface-}

This is of primary importance to standards bodies, such as CCA who want to make their standard be all SIDL interfaces, but currently have to make the exceptions SIDL classes because SIDL.BaseException is a class. This is an artifact that we unintentionally carried from Java and we plan to be correct it.

As of December 2003, there has been some initial design work. We have uncovered issues with Java bindings in response to this change that have yet to be resolved.

DONE. Released in Babel 0.9.0.

\section{Arrays}

\section{a. Increase max dimension from-4 to 7}

DONE. Released in Babel 0.8.8

\section{b. Array Base Classes (Added Oct 2004)}

Promoted from list of deferred items. Can also be seen as a SIDL issue. Users sometimes wish to specify an interface that takes arrays of arbitrary dimension or type. DONE (mostly). Expected in Babel 0.10.0 


\section{6. $C++$}

As of December 2003, Steven Parker from University of Utah has planned a January visit LLNL and work out an alternative $\mathrm{C}++$ binding to resolve the related outstanding issues. Changing the $\mathrm{C}^{++}$bindings is a BIG DEAL, but it is necessary to resolve some thorny issues, and clear a path for SCIRun to migrate from their C++-only PIDL tool (Parallel Interface Definition Language) to SIDL and Babel.

\section{a. Resolve issues with overloading based on object type}

Babel's C++ bindings predate Babel's overloading mechanism. The C++ stubs simulate the SIDL inheritance hierarchy, but do not implement it directly. The benefit is that method dispatch is slightly faster by circumventing $\mathrm{C}++$ vtables and going directly to Babel's EPVs. The downside is that once overloading was introduced, the C++ compiler doesn't have the right information to resolve the overloading.

\section{b. Resolve issues with exception handling of Babel smart- pointers}

This is another unfortunate side-effect of Babel's current $\mathrm{C}++$ stubs. The issue is that the $\mathrm{C}++$ code cannot catch parent classes of the SIDL declared exception, it must catch the exceptions listed in the SIDL specification exactly.

\section{Java}

As of December 2003, we now have Java bindings that support all the basie SIDL types (bool, char, int, float, long, double, fcomplex, deomplex, and strings), both client side and server side. However, arrays and objects remain outstanding issues. Arrays and Objects are historically the most challenging features of any language binding. The reasons for the delays in Java is that Fortran became a very big deal for our customers and Java was put on the back-burner.

\section{a. Implement Array support (client and server)}

Native Java arrays are not suitable for our purposes (appear to require copying data in all cases). Python had a similar problem, but there was a single weestablished array library (Numeric) that was a natural fit. We have yet to find asimilarly obvious choice for scientific arrays in Java.

\section{b. Implement support for Objects as arguments (client and server)}

There seems to be no outstanding technical issues to resolve, it is simply a matter of finding an available pool of effort to throw at the problem. 


\section{8. $F 90$}

\section{a. Provide native access to Babel arrays (a.k.a. Phase III)}

DONE. Released in Babel 0.8.8. Introduced new dependency on Chasm.

\section{b. Find solution for compilers that treat intrinsic functions as reserved words}

Some compilers treat intrinsic functions as reserved words. Although Babel does try to warn when reserved words in a particular language show up in a SIDL specification, we think prohibiting all the Fortran 90 intrinsic functions is a bit onerous. We hope to find a workaround before the Babel 1.0 release, but this issue will not be a blocker for the 1.0 release.

\section{Documentation}

\section{a. More detail on SIDL language}

We will add an annotated EBNF specification of the SIDL grammar and walk through more techniques about how to transfer favorite programming idioms from different languages to SIDL.

\section{b. More Examples}

Our Users' Guide has been described as encyclopedic and very dense. Users have asked for more examples to reduce the slope of the learning curve in the first couple sections.

\section{c. More Tutorials}

Babel has developed a half-day tutorial and delivered a dry-run to the CASC stmmer interns in 2003. We will be polishing the viewgraphs, presentations, and demonstrations for the 1.0 release.

DONE. Full day tutorial and hands-on to be presented at Supercomputing 2004

\section{Platforms}

\section{a. AIX using native $x \mid$ Compilers}

DONE (mostly). Official support announced in Babel 0.8.6. Unresolved issues with server-side python support.

\section{b. Revivify Solaris using gcc/SunF90}

DONE. Will be included in next release after Babel 0.8.8. 


\section{c. Linux64}

DONE (mostly). Works with Babel 0.8.9, but not instituted in nightly testing.

d. Mac OSX 


\section{Deferred Items}

The following items are frequently requested features. We know only that they are not planned for Babel 1.0. There's no telling if they will ever get into Babel for that matter, but we haven't removed them entirely from future considerations either.

1. Adding type hierarchy information to sidl.ClassInfo

2. Change from Numeric Python to numarray

3. Stop generating IOR.c files with client-side binding DONE. Expected in the 0.10 .0 release.

4. Typed Opaques

5. Array Base Class(es) (promoted to issue 5.b. August 2004) 